\title{
Concepciones y prácticas de profesores de Historia sobre la construcción didáctica del tiempo histórico mediado por el uso de las TIC
}

Concepts and practices of history teachers on the didactic construction of historical time mediated by the use of ICT

Recibido: 9 de octubre de 2019 | Aprobado: 25 de noviembre de 2019

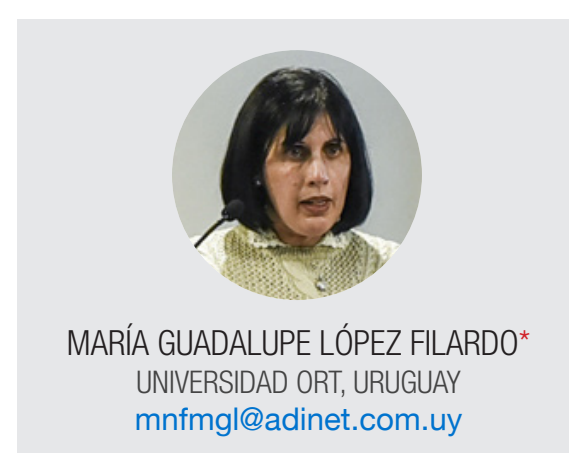

\section{Resumen}

Este artículo ${ }^{1}$ presenta las características y los resultados de un estudio llevado a cabo en el área de la formación inicial del profesorado en Uruguay². Su principal objetivo ha sido develar las concepciones de profesores de Historia sobre la enseñanza del tiempo histórico y la manera en que, de acuerdo con su formación y experiencia profesional, desarrollan prácticas de enseñanza mediante la integración y el uso de Tecnologías de la Información y Comunicación (TIC) con el fin de facilitar y mejorar la comprensión de dicho concepto. El diseño de esta investigación de corte cualitativo, basado en un estudio de casos múltiples, ha contado con la participación de un grupo de formadores de futuros docentes y otro de profesores noveles. Los resultados obtenidos tras la aplicación de una combinación de técnicas (entrevistas en profundidad, grupos focales, observaciones de clase y análisis de documentos) revelan que los sentidos y significados que los profesores atribuyen al uso de TIC en la enseñanza de conceptos históricos están determinados por sus concepciones epistemológicas, sujetas al campo disciplinar de formación y a sus experiencias docentes. Sus prácticas, fundadas en un sólido conocimiento disciplinar, si bien impulsan formas de pensar históricamente, no capitalizan la versatilidad de la tecnología digital para promover procesos innovadores en la enseñanza del tiempo histórico.

Palabras clave: didáctica; concepciones docentes; tiempo histórico; TIC; formación docente.

\footnotetext{
* Doctora en Educación por la Universidad ORT Uruguay. Master en Educación por la Universidad ORT Uruguay. Licenciada en Ciencias Históricas por la Universidad de la República. Es profesora de Teoría y Metodología de la Historia y de Historia de la Historiografía en el del Instituto de Profesores "Artigas", integrante del Consejo de Formación. Para contactar a la autora: mnfmg|@adinet.com.uy

1 El presente artículo forma parte de una tesis doctoral realizada en la Universidad ORT Uruguay de la misma autora.

2 La formación docente en Uruguay se halla organizada en torno a los tres niveles que conforman la educación básica, es decir: formación de maestros para la Educación Inicial y Primaria, de profesores para la Educación Media (conocida genéricamente bajo la denominación de Educación Secundaria) y de maestros técnicos para la Educación Técnico-Profesional. La formación inicial de profesores a la que refiere el presente artículo se realiza en función de los distintos campos disciplinares que comprende la Educación Media.
} 


\section{Abstract}

This paper ${ }^{3}$ shows the characteristics and results of a study carried out in the area of initial teacher training in Uruguay ${ }^{4}$. Its main objective has been to unveil the conceptions of history professors on the teaching of historical time, and the way in which, according to their training and professional experience, they develop teaching practices through the integration and use of Information and Communication Technologies (ICT) in order to facilitate and improve the understanding of this concept. The design of this qualitative research, based on a multiple case study, has been attended by a group of trainers of future teachers and another of novice teachers. The results obtained after the application of a combination of techniques (in-depth interviews, focus groups, class observations and document analysis) reveal that the senses and meanings that teachers attribute to the use of ICT in the teaching of historical concepts are determined by their epistemological conceptions, subject to the disciplinary field of training and their teaching experiences. Their practices, based on a solid disciplinary knowledge, while driving historical thinking, do not capitalize on the versatility of digital technology to promote innovative processes in the teaching of historical time.

Keywords: didactic; teaching conceptions; historical time; ICT; teacher training

\section{Introducción}

En la actual sociedad de la información y el conocimiento, el uso generalizado de tecnologías digitales, redes telemáticas y dispositivos móviles se ha convertido en "una de las señas identificatorias del presente" (Área Moreira, 2015, p. 177). Las Tecnologías de la Información y la Comunicación (en adelante $\mathrm{TIC}$ ), son percibidas como algo transparente que envuelve de manera imperceptible toda nuestra vida, impulsando nuevos alfabetismos y nuevas formas de comunicación y de acceso al conocimiento.

Sin embargo, al tiempo que el uso de la tecnología digital se expande y crece en forma exponencial en los distintos planos de la vida social, su incidencia en la cotidianidad de las aulas parecería ser todavía moderada. Este fenómeno ha dado lugar a una gran variedad de estudios e informes realizados desde diferentes ámbitos, por investigadores de campos disciplinares diversos, al punto de convertirse en una de las líneas relevantes en la investigación educativa actual y una de las metas prioritarias de las políticas educativas en la mayor parte de los países de la región y del mundo. En particular, los estudios sobre las concepciones de los profesores ${ }^{5}$ y el análisis de sus prácticas profesionales mediadas por el uso de TIC han contribuido a ampliar y profundizar el conocimiento en torno a las posibilidades que esta asociación es capaz de potenciar en la producción del saber. La investigación que hemos venido desarrollando en el marco de nuestra tesis doctoral, cuya síntesis global ofrecemos en el presente artículo, se inscribe precisamente dentro de esta línea de trabajos.

Originado en una serie de inquietudes que devienen de nuestra propia experiencia profesional en el campo de la enseñanza de la Historia, hemos buscado, a través de este estudio, conocer cómo conciben los profesores formados en esta disciplina el abordaje teórico y práctico de conceptos cuya comprensión es ineludible desde la lógica disciplinar, y en qué forma el uso de la tecnología digital contribuye a favorecer procesos de transformación de los saberes y prácticas docentes para facilitar y mejorar su enseñanza. De ahí la importancia de indagar - siguiendo la línea de lo planteado por Fullan (2012) - qué prácticas del profesorado se ven alteradas por la incorporación de las TIC; si

3 This article is part of our doctoral thesis conducted at the Universidad ORT Uruguay.

4 The teacher training in Uruguay is organized around the three levels that make up the basic education, that is: teacher training for Initial and Primary Education, teachers for Middle Education (known generically under the name of Secondary Education) and of technical teachers for Technical-Professional Education. The initial training of professors referred to in this article is carried out according to the different disciplinary fields included in Secondary Education.

5 En atención a principios de economía de lenguaje y a efectos de dar mayor fluidez a su lectura, en la redacción del presente artículo se ha optado por utilizar el genérico masculino en toda su extensión, sin que ello implique ninguna consideración discriminatoria o valoración peyorativa con relación al género femenino. 
se trata de alteraciones derivadas de la selección, incorporación y uso de recursos tecnológicos; de cambios en los enfoques y modelos didácticos desde los que se tiende a pensar otras formas de interacción con el conocimiento, o bien, de cambios en las concepciones docentes, es decir, en las presunciones bajo las cuales subyacen ciertas teorías sobre la enseñanza que coadyuvan a justificar y delimitar el empleo de la tecnología.

En los siguientes apartados trazamos una breve descripción del encuadre teórico en que se inscribe el estudio que hemos realizado, sus objetivos, los fundamentos y las particulares características de su diseño metodológico, y una síntesis global de los principales resultados obtenidos de la evidencia empírica analizada, así como también, algunas conclusiones que invitan a pensar nuevas formas de integrar la tecnología a las prácticas de enseñanza en la formación inicial del profesorado.

\section{Encuadre teórico de la investigación}

En las últimas décadas, la progresiva incorporación de las TIC en el contexto educativo ha ido imponiéndose en el discurso político y en el imaginario social como un indicador de innovación y mejora de la formación y el desarrollo profesional docente. En la mayoría de los países de la región las administraciones educativas han desplegado políticas orientadas a incentivar el desarrollo de prácticas de enseñanza sustentadas en el uso de tecnologías digitales, a fin de propiciar los cambios demandados por la sociedad del conocimiento. Tales esfuerzos, sin embargo, parecen haber resultado insuficientes para lograr que las TIC se configuren como herramientas cotidianas del trabajo escolar, al punto que cabe preguntarse, como señalan Vaillant y Marcelo (2015), “¿Por qué después de miles de acciones formativas que las distintas instituciones de formación continua de docentes han venido desarrollando (...) nos seguimos encontrando con el hecho de que el docente integra poco las tecnologías en sus procesos de enseñanza?" (p. 157).

Conforme revelan algunos estudios sobre las concepciones de los docentes en relación al uso de las TIC (Arancibia, Casanova y Soto, 2016; Báez y García, 2016), resulta paradójico que pese al discurso favorable que prima en general entre el profesorado respecto a los beneficios que aporta la incorporación de la tecnología en la enseñanza, no se observe una significativa transformación de sus prácticas. Ello podría obedecer, entre otras razones, a que las estructuras de pensamiento de muchos docentes continúan ancladas en paradigmas tradicionales, lo que hace que una innovación, como podría suponer el aprovechamiento de las TIC para la renovación de los métodos de enseñanza, termine siendo penosamente absorbida por arraigados modelos reproductores de prácticas que no responden a los nuevos enfoques de la didáctica disciplinar.

Un aspecto poco visible, pero de singular importancia para lograr un uso eficiente e innovador de las TIC en las prácticas de enseñanza, es el referido al conocimiento profesional docente, es decir, a los saberes, las habilidades y concepciones que van conformándose en el tránsito por la formación inicial y que se ven convalidadas en las experiencias generadas en el ejercicio de la profesión. La incorporación con sentido pedagógico de TIC (Dussel, 2015) depende, en gran medida, de las creencias y teorías implícitas que posee el profesorado sobre la enseñanza (Ertmer, 2005), derivadas de su formación, su experiencia y de la cultura institucional (Carbonell, 2006; Webb y Cox, 2004). Por ende, un elemento clave que facilita o dificulta la implicación de los docentes en proyectos de innovación con tecnologías es la compatibilidad entre sus creencias pedagógicas y la tecnología (Marcelo, 2013; Vaillant y Marcelo, 2015).

Hoy en día el uso de TIC con sentido pedagógico se ha convertido en uno de los mayores retos para los docentes, debido a las decisiones que deben tomar con respecto a cómo, cuándo, por qué y para qué integrarlas en la construcción del conocimiento. Un claro ejemplo de esta situación se plantea en la enseñanza de conceptos históricos que detentan un alto grado de abstracción, lo que demanda, además del saber pedagógico y disciplinar, el conocimiento tecno-pedagógico del contenido (Koehler, Mishra y Cain, 2015). Esto significa, que no es suficiente con que los profesores posean conocimientos sobre informática, sepan navegar por Internet, o utilizar distintos programas de productividad, puesto que tales habilidades les permitirán “(...) aprovechar las TIC de manera básica 
y generalista, pero no necesariamente serán útiles para abordar un contenido curricular específico de una asignatura, asociado con una aproximación pedagógica específica" (Hepp, 2015, p. 199).

Desde hace más de una década, las autoridades que conducen la formación inicial docente en Uruguay han impulsado, entre sus líneas estratégicas de trabajo, diversas iniciativas tendientes a fortalecer la formación en TIC de los futuros profesionales de la educación y la de sus formadores, con el fin de mejorar la enseñanza y los resultados de los aprendizajes. Sin embargo, pese a que tales proyectos se han visto fortalecidos con una sostenida política de equipamiento digital y conectividad proporcionada por el Plan Ceibal ${ }^{6}$, la esperada renovación de las prácticas de enseñanza aún no satisface las expectativas iniciales de la política educativa nacional (Caballero, 2017).

En lo referente al profesorado de Historia, existe una gama heterogénea de prácticas, experiencias, sentidos y cosmovisiones sobre la disciplina en general y sobre la enseñanza de conceptos fundamentales para la adquisición del conocimiento histórico. El tiempo histórico es uno de esos conceptos cuyo dominio exige el desarrollo de habilidades cognitivas que solo pueden ser producto de una enseñanza intencionada e inscrita en la línea del "pensar históricamente" (Gómez, Ortuño y Molina, 2014)

Más allá de los énfasis que puede conceder cada profesor a la enseñanza del tiempo histórico, existe consenso entre investigadores y didactas de la Historia en reconocer que este metaconcepto solo puede comprenderse en relación con los conceptos que lo integran y le dan sentido (Santisteban, 2007). Conceptos tales como cronología, periodización, cambio, continuidad, simultaneidad, duraciones, se adscriben al conjunto de nuevas ideas surgidas de la observación y de reflexiones emanadas de la propia naturaleza del conocimiento histórico, que se entienden como sinónimo de cambios en la agenda actual de la didáctica de la Historia. Habida cuenta de esto es importante saber, por ejemplo, qué representaciones sobre el tiempo histórico tienen los profesores de Historia; cuáles son las categorías temporales de amplio dominio y cuáles les resultan más difíciles de enseñar; qué modelos conceptuales y qué estrategias de intervención utilizan para favorecer en los estudiantes la comprensión de estos conceptos y, específicamente, cuáles son las formas de gestionar el conocimiento histórico que apelan al empleo eficiente e innovador de las TIC (Ertmer y Ottenbreit-Leftwich, 2010) lo que, consecuentemente, podría contribuir a modificar sus enfoques y propuestas de enseñanza.

\section{Definición del problema y objetivos}

Cabría tal vez preguntarse ¿por qué una investigación sobre las concepciones de los profesores acerca de la enseñanza del tiempo histórico en relación con el uso didáctico de TIC? Las razones que justifican la elección de esta temática tienen que ver, por una parte, con la pertinencia conceptual y metodológica que encierra el tiempo histórico, dado que su comprensión y la compleja representación de su dimensionalidad influyen de manera fundamental en multitud de decisiones relativas a su enseñanza. Y por otra, porque pese a la trascendencia que tiene la adquisición de nociones temporales para el desarrollo de habilidades en la interpretación del pasado, el conocimiento respecto a los procesos de formación del profesorado en este sentido, especialmente asociados al uso de la tecnología, ha sido un aspecto relegado en los escritos teóricos y las investigaciones referidas al tema.

Hace ya largo tiempo uno de los mayores exponentes en la didáctica del tiempo histórico sostenía que "La investigación educativa sobre el tiempo histórico se ha centrado mucho más en los resultados de aprendizaje que en el conocimiento de su enseñanza y de su aprendizaje en contextos concretos" (Pagès, 1999, p. 2). En efecto, a pesar de la centralidad disciplinar y curricular que reviste

6 Creado en el año 2007 en la órbita de la Presidencia de la República como iniciativa destinada a "promover la inclusión digital para un mayor y mejor acceso a la educación y la cultura" y sustentado en el modelo One Laptop per Chile (OLPC), el Plan de Conectividad Educativa de Informática Básica para el Aprendizaje en Línea (Ceibal), ha facilitado la incorporación masiva de herramientas digitales entre docentes y estudiantes, favorecido la creación y el desarrollo de ambientes con amplia disponibilidad tecnológica en los distintos niveles del sistema educativo. También ha asegurado el soporte de conectividad de los centros educativos de gestión estatal a escala nacional, a la vez que coordina y desarrolla programas de apoyo a las políticas educativas sobre inclusión digital. 
para el aprendizaje de la Historia el dominio de conceptos sobre la temporalidad histórica, son escasos los estudios llevados a cabo en el área de la formación inicial docente.

De acuerdo con lo señalado por Pagès y Santiesteban (2013), la información sobre cómo se forman los profesores para responder a los desafíos que implica la enseñanza del tiempo histórico en los distintos niveles educativos es aún insuficiente. Buena parte de la literatura sobre estas cuestiones aparece dominada por la identificación de estrategias o de innovaciones puntuales que han resultado exitosas, pero la dimensión menos explorada es la que concierne concretamente a la inclusión de TIC en la enseñanza de este metaconcepto. Es preciso, por lo tanto, disponer de más investigación que permita conocer cuáles son las concepciones que ha ido construyendo el profesorado en los distintos trayectos de su formación y que orientan sus intervenciones didácticas, cómo son trabajados en la cotidianeidad de las aulas los conceptos de tiempo histórico y cómo incide la integración de tecnologías digitales en las prácticas de enseñanza enfocadas a la comprensión y el dominio de las distintas dimensiones temporales.

Los aspectos específicos vinculados al propio concepto de tiempo histórico (su dominio conceptual y didáctico) y los aspectos referidos a la integración y el uso de las TIC en la construcción del conocimiento histórico representan la problemática de este estudio. Ello implica conocer las representaciones de los profesores sobre el tiempo histórico, saber cómo elaboran y llevan a la práctica secuencias didácticas para trabajar estos conceptos y, en particular, qué recursos tecnológicos utilizan y de qué modo.

Ha sido precisamente la búsqueda de respuestas a estas interrogantes lo que ha impulsado la realización de un estudio empírico de características descriptivas, analíticas e interpretativas, que conjuga diversos componentes.

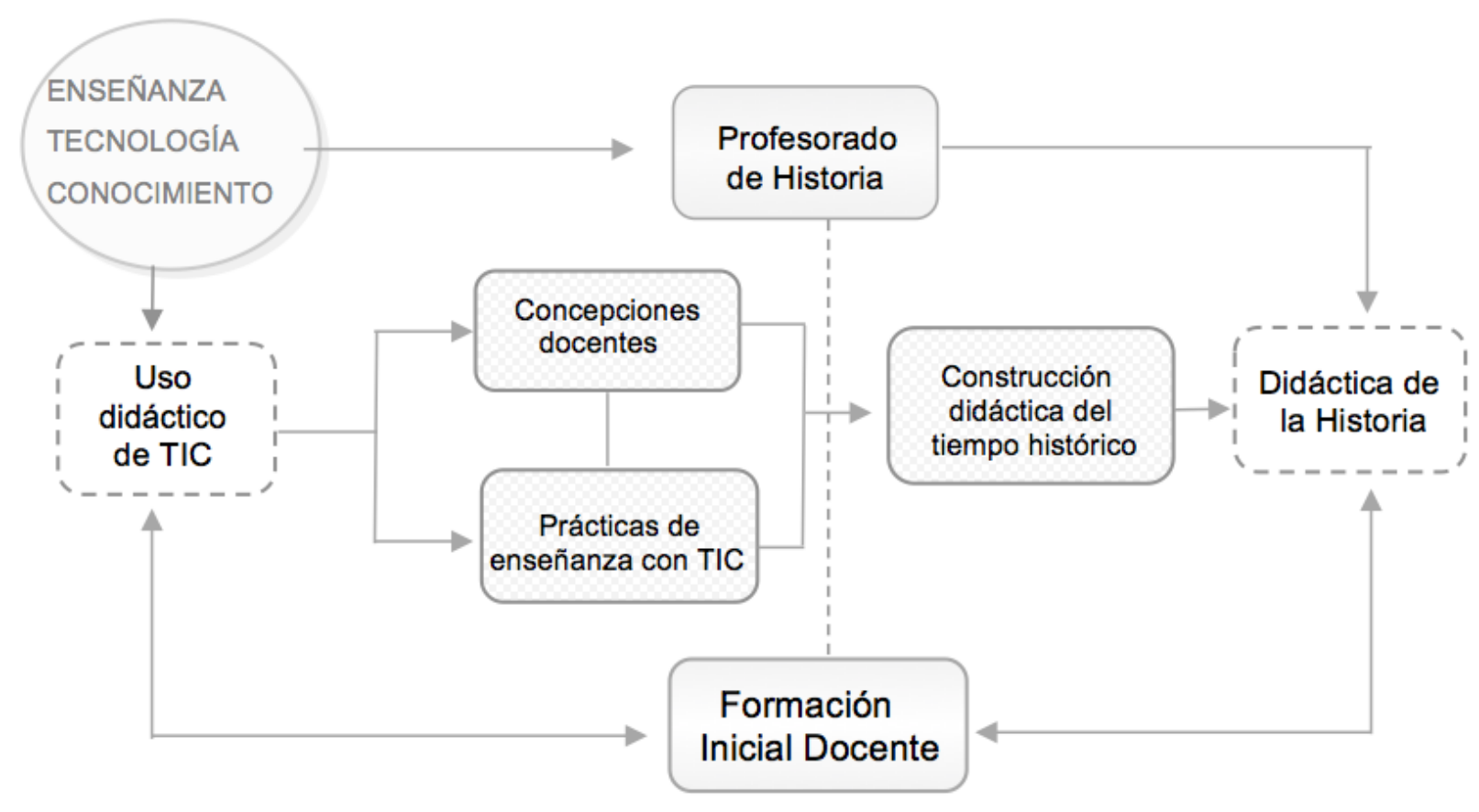

Figura 1. Componentes de la investigación 
El principal objetivo del estudio ha sido analizar las concepciones de los profesores de Historia sobre la construcción didáctica del tiempo histórico y la integración curricular que hacen de las TIC para lograrlo.

Los propósitos específicos fueron:

- Indagar las concepciones que, en función de su formación y experiencia profesional, posee el profesorado de Historia acerca del tiempo histórico, su enseñanza, y del uso de las TIC para una mejor comprensión y dominio del mismo.

- Identificar los recursos tecnológicos utilizados por profesores de Historia al implementar secuencias didácticas destinadas a la enseñanza del tiempo histórico y analizar la pertinencia de su selección y adecuación pedagógica.

- Caracterizar los modos en que los formadores de profesores y los profesores principiantes integran las TIC en la construcción didáctica del tiempo histórico.

\section{Diseño metodológico}

El esfuerzo metodológico de esta investigación, centrado en estudiar el fenómeno descrito anteriormente, se tradujo en el empleo de una metodología cualitativa, que combina enfoques fenomenológico y hermenéutico, con énfasis en la etnografía. La opción por una lógica investigativa anclada en el paradigma cualitativo (Cook y Reichardt, 2000) respondió a la necesidad de someter a un riguroso análisis inductivo una realidad educativa compleja, que no cabe considerar en términos de cantidad, intensidad o frecuencia, sino percibirla e interpretarla desde la óptica de los significados subjetivos que le atribuyen las personas (Denzin y Lincoln, 2012).

Como estrategia metodológica se apeló a un estudio de casos de tipo múltiple, o "estudio colectivo" según Stake (2010), a través del cual fueron analizados en forma intensiva y simultánea doce casos, considerándose cada profesor un caso en sí mismo y una fuente de información especializada, de conformidad con los propósitos de la investigación. Las unidades de observación estuvieron representadas por los profesores, en tanto que las unidades de análisis fueron, por un lado, sus concepciones sobre la enseñanza del tiempo histórico, y por otro, sus prácticas de enseñanza mediadas por el uso de TIC.

\section{Población y contexto de la investigación}

La selección de las unidades de observación fue realizada aplicando el principio de "muestreo teórico" sugerido por Mejía Navarrete (2000) en la investigación cualitativa. Para ello partimos de algunos criterios establecidos a priori, con el objeto de lograr la participación de un conjunto diverso y heterogéneo de casos (profesores vinculados al área de la formación inicial del profesorado de Historia), a modo de obtener una mirada múltiple y a la vez interconectada. Como puede apreciarse en la siguiente tabla, la muestra resultante, de carácter intencional, comprendió dos "estamentos" (Cisterna, 2005) de docentes, uno integrado por seis formadores - quienes a los efectos del presente estudio fueron identificados con la denominación de "expertos" - y el otro, por seis profesores "noveles".

Tabla 1. Composición de las unidades de observación

\section{EXPERTOS}

Profesores de Didáctica de la Historia, quienes a través de actividades teórico-reflexivas en el campo disciplinar específico, orientan la epistemología de la práctica guiando las intervenciones de estudiantes que cursan la carrera.

Profesores de Historia de reconocida actuación en Educación Secundaria que actúan en calidad de profesores adscriptores (tutores) de estudiantes que realizan la práctica docente correspondiente a los primeros cursos de profesorado.

\section{NOVELES}

Profesores de Historia recientemente egresados, que transitan el primer trienio de ejercicio profesional desempeñando funciones docentes en centros de Educación Secundaria, y que conservan vínculos con referentes de su formación inicial.

Estudiantes-practicantes de Historia que, cursando la didáctica-práctica correspondiente al último año de la carrera, actúan en calidad de profesores de un grupo de estudiantes de Educación Secundaria, con la orientación y supervisión del profesor de Didáctica. 
Además de las características particulares señaladas en la tabla precedente, se contemplaron dos requisitos comunes a todos los participantes. Por un lado, a efectos de asegurar accesibilidad a las fuentes (Valles, 2003), se seleccionaron profesores que ejercen funciones en centros educativos ubicados en la misma ciudad, y por otro, se tuvo en cuenta solo a aquellos profesores dispuestos a participar voluntariamente en el estudio. En función de este último criterio, antes de iniciar el trabajo de campo, se propuso a cada profesor la suscripción de un documento de consentimiento informado, en el que debían expresar su conformidad y compromiso de participación.

La investigación se desarrolló durante el primer y segundo semestre del año lectivo 2017 en dos espacios académicos ligados por una red de relaciones sostenidas por el rol que cumplen distintos actores: el centro de formación inicial de profesores, que nuclea a integrantes de ambos estamentos (formadores y profesores recién egresados o en vías de próximo egreso), y los centros de Educación Media (liceos), en que desempeñan tareas docentes los profesores adscriptores (tutores) y los noveles profesores.

En tanto estructura lógica, el diseño de la investigación contiene distintos componentes cuya disposición y coherencia interna se hallan representadas en el siguiente diagrama. E empleo de conectores indicando las relaciones entre los mismos, pone de relieve "una estructura interconectada y flexible" (Maxwell, 1996, p. 2).

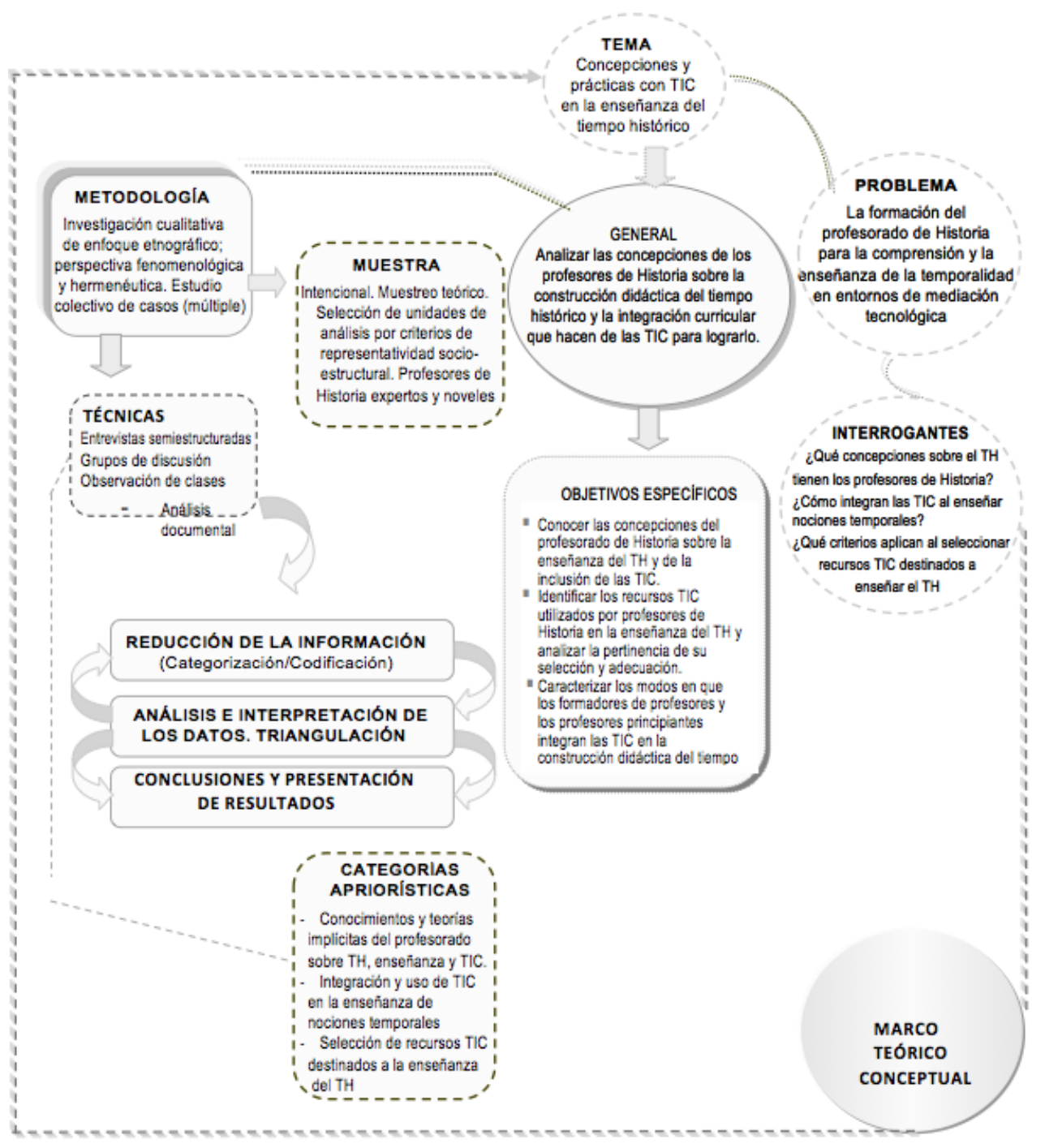

Figura 2. Estructura general del diseño de investigación 
Técnicas e instrumentos de recogida de la información

La amplitud y complejidad de la información requerida para cumplir con los objetivos del estudio obligó a pensar en una combinación de técnicas con diferente grado de interrelación (Flick, 2007) como estrategia para obtener datos relevantes, conducentes a la construcción de un conocimiento más holístico y comprensivo del problema. En consecuencia, se realizaron: entrevistas en profundidad, grupos de discusión, observaciones de clases y análisis de documentación docente.

En el caso de las entrevistas, se optó por la semiestructurada, basado en preguntas presentadas a través de un diálogo abierto y flexible, para facilitar la libre expresión de respuestas de cada entrevistado y obtener una mejor calidad de conocimiento (Kvale, 2011). Para su conducción se diseñó una pauta de carácter orientativo (Flick, 2007), compuesta por una serie de interrogantes organizadas en torno a las tres grandes dimensiones de análisis construidas teóricamente: (i) identificación del perfil personal, profesional y nivel de competencias tecnológicas de cada entrevistado, (ii) sus concepciones respecto a la enseñanza del tiempo histórico y la tecnología digital en los procesos de enseñanza y aprendizaje, (iii) los usos de las TIC en la construcción didáctica de conceptos sobre la temporalidad histórica.

La información recogida en las entrevistas individuales se complementó con la obtenida en dos grupos de discusión: uno entre profesores expertos y otro entre noveles. Aprovechando la sinergia generada por el intercambio, la motivación y naturalidad que supone la propia dinámica grupal, los profesores pudieron exponer visiones derivadas de sus experiencias personales con respecto a la enseñanza del tiempo histórico y la inclusión de TIC en sus prácticas profesionales. El uso de esta técnica resultó especialmente pertinente para comprender, como sugiere Barbour (2013), cómo las personas elaboran socialmente un conocimiento crítico del tema planteado. El instrumento utilizado para ordenar la información de ambos grupos de discusión fue una guía en la que, además de explicitarse los objetivos de la deliberación, se establecieron pautas relativas a la dinámica de trabajo y al rol de observador/moderador que en esta instancia asumimos personalmente. Durante el desarrollo de las sesiones se plantearon preguntas referidas a los dos grandes focos de indagación del estudio: (i) el tiempo histórico y su enseñanza, (ii) la selección, evaluación y el uso didáctico de recursos tecnológicos para la comprensión y el dominio de nociones temporales.

Una tercera técnica fue la observación de clases con el propósito de recoger información acerca de la forma en que profesores de Historia desarrollan secuencias didácticas destinadas a la enseñanza del tiempo histórico con la mediación de TIC. En función de los objetivos de la investigación, se realizó un tipo de observación directa, al tiempo que, para asegurar distanciamiento respecto de la realidad observada, optamos por la modalidad de observación no participante (Yuni y Urbano, 2006). El instrumento diseñado para el registro de las observaciones fue una guía semi-cerrada y adaptada a las demandas particulares de cada observación. Su estructura, organizada en siete secciones, permitió recoger datos relativos al proceso de desarrollo de cada secuencia y sus distintos componentes.

A efectos de complementar y contrastar la información obtenida con estas técnicas y poder "describir y enriquecer el contexto y contribuir al análisis de los temas" (Simons, 2011, p. 97), se examinó una muestra de documentos producidos por los profesores participantes en la investigación sobre la integración de TIC en la enseñanza del tiempo histórico. El instrumento utilizado para registrar el análisis de dichos documentos consistió en una ficha construida con base en un conjunto de indicadores, organizados en distintas dimensiones y sub-dimensiones, a fin de identificar el diseño de los respectivos materiales y las formas en que son explicitadas las dinámicas de trabajo y de uso de los recursos TIC.

\section{Criterios de validez y rigor científico}

Con el fin de dotar de validez y fiabilidad a los instrumentos diseñados para la recogida de información, se los sometió a una cuidadosa revisión (Mejía, 2011), adoptándose dos tipos de estrategias: los instrumentos de entrevista (individual y grupal) fueron validados mediante juicio de expertos, mientras que los instrumentos destinados al registro de observaciones de clase 
y análisis documental fueron testeados a través de sucesivas aplicaciones en forma piloto. Para la validación de los instrumentos de entrevista se contó con la colaboración de tres especialistas de amplia trayectoria profesional en el ámbito académico nacional, poseedores de una reconocida producción intelectual y de investigación en las áreas en que se enmarca el estudio. Estos jueces fueron seleccionados mediante el procedimiento de biograma (Cabero y Llorente, 2013) y sus aportes permitieron ajustar los instrumentos a su versión definitiva.

Entre los criterios adoptados para asegurar autenticidad al proceso de indagación se tuvo en cuenta, entre otros, la revisión por parte de los profesores de las transcripciones de las entrevistas, con el fin de salvaguardar la exactitud de lo expresado en ellas. Además, en el análisis de los datos se consideraron especialmente los criterios de credibilidad, transferibilidad, dependencia y confirmabilidad propuestos por Guba y Lincoln (2012), matizados a su vez por la práctica de la reflexividad, para evitar que las predisposiciones personales pudieran afectar el proceso de investigación y sus resultados. Se aplicó también el criterio de multiplicidad de voces (Moral, 2006), otorgando un lugar preferencial en el informe a las voces de los informantes.

La otra alternativa de validación empleada con objeto de garantizar el control de calidad del proceso de investigación fue la triangulación hermenéutica (Cisterna, 2005). Con base en los datos recogidos de las distintas fuentes, se realizaron dos instancias de triangulación, una de primer orden, considerando las fuentes primarias de datos, y luego una triangulación de segundo orden, a efectos de generar nuevos procesos interpretativos y verificar posibles resultados similares (Flick, 2014).

\section{Resultados}

A partir de los datos registrados en la primera parte de las entrevistas, se obtuvo el perfil socioprofesional de los participantes de la investigación. El grupo de expertos, mayoritariamente de género femenino, estuvo compuesto por seis docentes (tres profesores de didáctica y tres adscriptores), cuyas edades promedian los cincuenta años. Su formación se ha mantenido en permanente actualización a través de cursos de postgrado y su actuación docente supera en general los veinticinco años de servicio. En el grupo de noveles, integrado por tres profesores recién egresados y tres practicantes, se aprecia una representación proporcional de ambos géneros. Sus edades fluctúan entre los 21 y 28 años. Con respecto a su formación, dos de los recién egresados están cursando postgrados. Vale decir que los distintos niveles de formación y las respectivas trayectorias profesionales se hallan en consonancia con la conformación etaria de ambos grupos.

Con relación al uso de tecnologías digitales, si bien ninguno de los entrevistados se desenvuelve profesionalmente en esta área, manifestaron estar familiarizados y poseer experiencia en el empleo de dispositivos tecnológicos de uso común y conocer algunas aplicaciones informáticas que les permiten producir y publicar en distintos soportes. En cuanto a su formación en TIC, los expertos indicaron haber participado en instancias formales de capacitación, en tanto que, para los noveles, sus experiencias formativas han resultado insuficientes. A efectos de contrastar esta información con sus percepciones respecto al dominio de TIC para la función docente, se solicitó a cada profesor que definiera el lugar en que se ubicaría dentro de una escala comprendida en tres niveles: básico, avanzado y experto. Dos de los formadores se ubicaron en nivel básico, tres en avanzado y uno experto. Entre los noveles, los tres recién egresados se ubicaron en nivel avanzado, mientras que la generación más joven, es decir, quienes están culminando la etapa de formación inicial, a la vez que se auto-percibe en nivel básico, expresa necesidades de cara a la integración curricular de la tecnología en su quehacer docente.

El análisis cualitativo del material recogido se basó en un enfoque interpretativo (Taylor y Bodgan, 1990; Miles y Huberman, 1994). A partir de los datos procedentes de cada una de las técnicas aplicadas, se realizaron inferencias inductivas y se identificaron recurrencias, discordancias, regularidades, particularidades, sincronías y asincronías, a la luz del 
proceso de categorización realizado con base en las tres grandes dimensiones construidas teóricamente. Ello permitió establecer relaciones entre los casos y una posterior triangulación de resultados en función de los objetivos específicos de la investigación, según se detalla en la siguiente tabla.

Tabla 2. Síntesis de resultados según objetivos

\begin{tabular}{|c|c|c|}
\hline OBJETIVOS & DIMENSIONES & RESULTADOS (R) \\
\hline $\begin{array}{l}\text { Objetivo } 1 \\
\text { Indagar las concep- } \\
\text { ciones que, en función } \\
\text { de su formación y ex- } \\
\text { periencia profesional, } \\
\text { posee el profesorado } \\
\text { de Historia acerca del } \\
\text { tiempo histórico, su } \\
\text { enseñanza, y de las } \\
\text { TIC para una mejor } \\
\text { comprensión y dominio } \\
\text { del mismo. }\end{array}$ & $\begin{array}{l}1^{\text {a }} \text { dimensión } \\
\text { Concepciones de } \\
\text { los profesores de } \\
\text { Historia sobre la } \\
\text { enseñanza del } \\
\text { tiempo histórico y } \\
\text { la significación de } \\
\text { las TIC para mejorar } \\
\text { la comprensión de } \\
\text { este concepto. }\end{array}$ & $\begin{array}{l}\text { R1. Los profesores (expertos y noveles) conciben la construcción didáctica del conocimiento } \\
\text { histórico con base en el desarrollo de habilidades de pensamiento histórico. } \\
\text { R2. La enseñanza del tiempo histórico que desarrollan ambos estamentos se fundamenta, } \\
\text { principalmente, en el sentido de continuidad y cambio entre pasado y presente. } \\
\text { R3. Cronología, duraciones, relaciones de cambio y continuidad, y vínculos entre hechos históricos } \\
\text { y factores coyunturales, ocupan un lugar preferente en las secuencias didácticas sobre las } \\
\text { dimensiones del tiempo histórico que diseñan expertos y noveles. } \\
\text { R4. Los profesores expertos perciben más claramente las dificultades que entraña la comprensión } \\
\text { de conceptos temporales, particularmente en las prácticas desarrolladas en los procesos de } \\
\text { formación. } \\
\text { R5. Expertos y noveles entienden que el dominio de conceptos sobre la temporalidad histórica no } \\
\text { depende únicamente del nivel de desarrollo cognitivo de los estudiantes, sino de la forma en que } \\
\text { son enseñados. } \\
\text { R6. El sentido epistemológico y cultural que caracteriza las concepciones de los profesores } \\
\text { (expertos y noveles) sobre la integración de TIC en sus intervenciones didácticas se sustenta en } \\
\text { las potencialidades de estas para favorecer la interacción con el conocimiento disciplinar. } \\
\text { R7. Las TIC son percibidas como elementos mediadores en la comunicación y la comprensión de } \\
\text { reconstrucciones del pasado. } \\
\text { R8. La capacitación en TIC ofertada por el sistema formal de formación docente proporciona } \\
\text { herramientas para su dominio básico, pero no satisface las expectativas del profesorado novel en } \\
\text { cuanto a orientaciones pedagógicas y disciplinares específicas. }\end{array}$ \\
\hline $\begin{array}{c}\text { Objetivo } 2 \\
\text { Identificar los recursos } \\
\text { tecnológicos utilizados } \\
\text { por profesores de } \\
\text { Historia al implementar } \\
\text { secuencias didácticas } \\
\text { destinadas a la } \\
\text { enseñanza del tiempo } \\
\text { histórico y analizar } \\
\text { la pertinencia de su } \\
\text { selección y adecuación } \\
\text { pedagógica. }\end{array}$ & $\begin{array}{c}2^{a} \text { dimensión } \\
\text { Selección, creación } \\
\text { y evaluación } \\
\text { de recursos } \\
\text { tecnológico-digitales } \\
\text { destinados a la } \\
\text { enseñanza del } \\
\text { tiempo histórico. }\end{array}$ & $\begin{array}{l}\text { R9. Las principales motivaciones del profesorado (experto y novel) al seleccionar recursos TIC } \\
\text { están referidas a su adecuación a metas pedagógicas y a sus potencialidades para mejorar los } \\
\text { niveles de comprensión. } \\
\text { R10. Entre los objetivos que definen la selección de recursos TIC por parte de los profesores tienen } \\
\text { relevancia los contenidos curriculares, las estrategias de enseñanza y las formas de aprendizaje } \\
\text { de los estudiantes. } \\
\text { R11. La selección de recursos TIC destinados a la enseñanza del tiempo histórico que realizan, } \\
\text { especialmente los profesores noveles, se basa en razones de: funcionalidad, practicidad, } \\
\text { adecuación a la propuesta curricular y calidad del material. } \\
\text { R12. Las fuentes a las que acuden los profesores (expertos y noveles) para la selección, creación } \\
\text { y/o adaptación de recursos TIC son comúnmente materiales multimedia digitalizados y editables, } \\
\text { extraídos de repositorios educativos abiertos. } \\
\text { R13. Las características multimedia de las TIC para la representación de contenidos históricos son } \\
\text { valoradas positivamente por el profesorado en general. } \\
\text { R14. Los recursos tecnológicos digitales específicos para la enseñanza del tiempo histórico son de } \\
\text { escaso uso entre los profesores (expertos y noveles). } \\
\text { R15. Los criterios de selección de recursos TIC empleados por el profesorado novel obedecen } \\
\text { comúnmente a facilidades de acceso, localización, o a recomendaciones de colegas. } \\
\text { R16. La capacitación en el uso de TIC no contempla el análisis y la evaluación de la funcionalidady } \\
\text { calidad didáctica de los recursos empleados en la enseñanza de conceptos históricos, dependiendo } \\
\text { de la iniciativa y experiencia personal de cada profesor. }\end{array}$ \\
\hline
\end{tabular}




\begin{tabular}{|c|c|c|}
\hline $\begin{array}{c}\text { Objetivo } 3 \\
\text { Caracterizar los modos } \\
\text { en que los formadores } \\
\text { de profesores y } \\
\text { los profesores } \\
\text { principiantes } \\
\text { integran las TIC en la } \\
\text { construcción didáctica } \\
\text { del tiempo histórico. }\end{array}$ & $\begin{array}{c}3^{\text {a }} \text { dimensión } \\
\text { Integración y uso } \\
\text { de las TIC en la } \\
\text { enseñanza y el } \\
\text { aprendizaje de } \\
\text { nociones sobre } \\
\text { la temporalidad } \\
\text { histórica. }\end{array}$ & $\begin{array}{l}\text { R17. La integración de TIC en las propuestas de aula afecta positivamente la comunicación entre } \\
\text { profesores y estudiantes y fortalece la interacción entre estos y el conocimiento. } \\
\text { R18. En los documentos producidos por los profesores (expertos y noveles), la construcción } \\
\text { didáctica de conceptos de tiempo histórico tiene carácter informativo y operativo, pero carece de } \\
\text { explicitación respecto al uso de recursos tecnológicos. } \\
\text { R19. En las orientaciones que los expertos brindan a sus estudiantes sobre la enseñanza de } \\
\text { nociones temporales se omite generalmente la inclusión de TIC. } \\
\text { R20. Los recursos TIC más reconocidos por los profesores (expertos y noveles) para facilitar la } \\
\text { representación de categorías de tiempo histórico son: organizadores gráficos, imágenes, ejes } \\
\text { cronológicos, líneas de tiempo y mapas conceptuales. } \\
\text { R21. Las actividades de aprendizaje más frecuentes en las secuencias didácticas remiten a } \\
\text { búsqueda y organización de información, representación de contenidos, ejercitación y/o aplicación } \\
\text { de conocimientos, presentación de productos, requiriendo del uso de dispositivos electrónicos (no } \\
\text { siempre utilizados dentro del aula). } \\
\text { R22. Los procesos de evaluación en las secuencias didácticas suelen ser de carácter formativo, } \\
\text { pero no se reconocen estrategias específicas para analizar la comprensión de conceptos de } \\
\text { temporalidad histórica. } \\
\text { R23. La mayor debilidad en las propuestas del profesorado (experto y novel) tiene que ver con el } \\
\text { diseño de actividades que integren recursos tecnológico-digitales destinados a la enseñanza del } \\
\text { tiempo histórico. } \\
\text { R24. El enfoque que subyace al diseño de los recursos TIC que acostumbran emplear los } \\
\text { profesores (expertos y noveles) en sus clases es preferentemente cognitivo o constructivista, } \\
\text { aunque entienden que lo que prevalece es la forma en que se usan. }\end{array}$ \\
\hline
\end{tabular}

Los resultados relacionados con la primera dimensión de análisis dan cuenta de que:

- las concepciones de los profesores con respecto a la construcción del conocimiento histórico se sustentan en una enseñanza que problematiza el saber y fomenta el pensamiento histórico;

- las representaciones que sobre el tiempo histórico posee el profesorado de Historia determinan la manera en que es asumida la complejidad conceptual de su enseñanza;

- las estructuras temporales que han ido construyendo los profesores en los sucesivos trayectos formativos requieren de un andamiaje conceptual y práctico que va completándose en la formación inicial, pero que a menudo no concluye durante esta etapa, lo que hace necesario el empleo de estrategias didácticas que refuercen el desarrollo de habilidades en la interpretación del pasado;

- la valoración manifiesta en los discursos de los profesores acerca de los aportes de las tecnologías digitales para potenciar procesos metacognitivos y favorecer nuevas formas de aproximación al conocimiento histórico no se ve reflejada en la creación de ambientes de aprendizaje que frecuenten la inclusión y el uso de TIC en la enseñanza de conceptos históricos;

- el nivel de competencias tecno-pedagógicas que exhibe el profesorado en sus prácticas profesionales es en general limitado, e incluso presenta carencias en cuanto a la forma de definir el uso instrumental y didáctico de los recursos tecnológicos en el aula.

Con respecto a la segunda dimensión, los resultados obtenidos señalan que las decisiones que adoptan los profesores al seleccionar las TIC están basadas fundamentalmente en iniciativas personales o derivadas de su experiencia docente, lo que pone de relieve la necesidad de:

- revisar los criterios de selección de recursos TIC que utilizan los profesores en la enseñanza de contenidos históricos; 
- repensar las formas de creación y adaptación de recursos tecnológicos especialmente diseñados para facilitar la adquisición de nociones temporales;

- reflexionar acerca de cómo estimular el uso innovador de las tecnologías digitales en las prácticas de formación del profesorado.

Por último, los resultados derivados de la tercera dimensión de análisis nos permiten caracterizar el modo en que los profesores conciben la inclusión de las TIC en sus prácticas, según tres enfoques que hemos denominado: comprensivo, instrumental y estratégico.

Tabla 3. Caracterización de concepciones sobre integración de TIC en la enseñanza de conceptos temporales

\begin{tabular}{|c|c|}
\hline $\begin{array}{c}\text { Enfoque } \\
\text { comprensivo }\end{array}$ & $\begin{array}{l}\text { Los profesores manifiestan estar } \\
\text { convencidos de que las TIC cooperan } \\
\text { en la comprensión de conceptos } \\
\text { temporales y, en consecuencia, } \\
\text { estimulan al alumnado para que } \\
\text { establezca relaciones y otorgue } \\
\text { sentido al aprendizaje de los mismos. }\end{array}$ \\
\hline $\begin{array}{l}\text { Enfoque } \\
\text { instrumental }\end{array}$ & $\begin{array}{l}\text { Los profesores asumen las TIC } \\
\text { como herramientas eficaces para la } \\
\text { enseñanza de contenidos históricos, } \\
\text { por lo que consideran necesario su uso } \\
\text { para proporcionar a los estudiantes } \\
\text { experiencias de aprendizaje que } \\
\text { favorezcan el reconocimiento y la } \\
\text { apropiación de conceptos de tiempo } \\
\text { histórico. }\end{array}$ \\
\hline $\begin{array}{c}\text { Enfoque } \\
\text { estratégico }\end{array}$ & $\begin{array}{l}\text { Los profesores estiman que el uso } \\
\text { didáctico de las TIC se sustenta en el } \\
\text { conocimiento tecno-pedagógico del } \\
\text { contenido, indispensable para facilitar } \\
\text { la representación y el manejo de } \\
\text { nociones temporales. }\end{array}$ \\
\hline
\end{tabular}

Si bien estos enfoques no son neutros, es posible percibir en las intervenciones didácticas del profesorado de Historia:

- las motivaciones que definen la integración de las TIC en la enseñanza y el aprendizaje de conceptos históricos;

- la necesidad de una orientación específica de los formadores hacia el profesorado novel o en formación, sobre las distintas formas de inclusión de TIC en la construcción didáctica de conceptos de tiempo histórico;

- la importancia de que expertos y noveles posean conocimiento tecno-pedagógico del contenido, para poder seleccionar, crear y evaluar recursos TIC que ayuden a mejorar la comprensión y el dominio de conceptos temporales.

\section{Conclusiones}

De acuerdo con las evidencias recogidas y en convergencia con el marco teórico de la investigación, es posible concluir que las concepciones de los profesores sobre la naturaleza del conocimiento histórico y la lógica disciplinar, ejercen una decisiva influencia en la manera de entender y diseñar la enseñanza, a la vez que definen las formas en que significan y emplean la tecnología.

El conocimiento construido por los profesores de Historia acerca de los conceptos de tiempo histórico se relaciona, desde el punto de vista didáctico, con la forma en que diseñan estrategias para favorecer procesos comprensivos en la disciplina. Tales procesos no contemplan, sin embargo, la dinámica relación entre el uso pedagógico de las TIC y la construcción didáctica de conceptos estructurantes del saber disciplinar, lo que marca la necesidad de desarrollar, en los distintos trayectos de formación del profesorado, una sistematización de reflexiones teóricas y experiencias que promuevan una activa relación entre didáctica y tecnología.

Lo que define el modo en que las TIC se integran a las prácticas que desarrollan los profesores al construir nociones de tiempo histórico es el uso didáctico que hacen de las mismas y las 
interacciones que se promueven, las cuales están sujetas a sus intencionalidades pedagógicas, al dominio de competencias tecno-pedagógicas y al rol asumido por cada uno en la conducción de las prácticas de enseñanza. Mientras algunos profesores mantienen una acción reguladora con respecto a las actuaciones de sus alumnos, otros adoptan una posición más flexible, propiciando la participación autónoma y reflexiva de los estudiantes en las diversas actividades de aprendizaje. Estas diferentes posturas dependen del modelo pedagógico prevalente en cada docente, por lo que, en este sentido, muchas veces las prácticas con TIC se asemejan a las que se realizan sin el concurso de estas herramientas.

Si bien la principal motivación de los profesores al integrar recursos tecnológicos en la enseñanza de conceptos temporales es contribuir a facilitar la comprensión de los mismos, en el diseño de secuencias didácticas prevalecen los usos comunicativos por sobre los creativos, y no se establecen mecanismos de evaluación que permitan identificar el nivel de comprensión alcanzado por los estudiantes, ni la incidencia de las TIC en dichas operaciones. Será preciso, en tal sentido, continuar investigando las razones por las que, pese al explícito reconocimiento y valoración de las TIC en la enseñanza y el aprendizaje de conceptos históricos, es escasa la utilización de tecnologías digitales con sentido formativo que ayuden al profesorado a familiarizarse, superar dificultades de comprensión y establecer nuevas formas de relación con el conocimiento.

Creemos que los aportes empíricos de investigaciones de este tipo, además de contribuir a un mayor conocimiento y comprensión de las relaciones entre el uso pedagógico de la tecnología y la práctica profesional docente asociada a la enseñanza de contenidos disciplinares específicos, puede propiciar el acercamiento a una didáctica de la Historia preocupada por recuperar la dimensión relacional de lo que Fullan y Donnelly (2013) han definido como "nuevas pedagogías", capaces de impulsar caminos alternativos para generar prácticas de enseñanza verdaderamente innovadoras.

\section{Referencias}

Arancibia, M., Casanova, R. y Soto, C. (2016). Concepciones de profesores sobre aprender y enseñar usando tecnologías. Ciencia, Docencia y tecnología, 27(52), 106-126. Recuperado de http://pcient.uner.edu.ar/ index.php/cdyt/article/ view/162/198

Área Moreira, M. (2015). Reinventar la escuela en la sociedad digital. Del aprender repitiendo al aprender creando. En Poggi, M. (Coord.). Mejorar los aprendizajes en la enseñanza obligatoria: Políticas y actores. IIPE/UNESCO (pp. 167-194). Recuperado de http://unesdoc. unesco.org/images/0023/002349/234977s. pdf

Báez, M. y García, J.M. (Comps.) (2016). Reflexiones (y provocaciones) acerca del lugar de las TIC en la formación docente. En Educación y tecnologías en perspectiva. 10 años de FLACSO Uruguay (pp. 13-28). Recuperado de http://flacso.edu.uy/publicaciones/libro_ educacion_tecnologia_2016/Baez_Garcia_TIC_ en_formacion_docente.pdf

Barbour, R. (2013). Los grupos de discusión en Investigación Cualitativa. Madrid: Morata.

Cabero, J. y Llorente, M.C. (2013). La aplicación del juicio de experto como técnica de evaluación de las tecnologías de la información (TIC). Revista de Tecnología de Información y Comunicación en Educación, 7(2), 11-22. Recuperado de http://servicio.bc.uc.edu.ve/ educacion/eduweb/v7n2/art01.pdf

Caballero de Luis, S. (2017). Una revisión sistemática a 10 años del Plan Ceibal en Uruguay. Didáskomai, 8, 28-44.

Carbonell, J. (2006). La aventura de innovar. El cambio en la escuela. Madrid: Morata.

Cisterna Cabrera, F. (2005). Categorización y triangulación como procesos de validación del conocimiento en investigación cualitativa. Theoria, 14(1), 61-71. Recuperado de http:// www.ubiobio.cl/theoria/v/v14/a6.pdf 
Cook, T.D. y Reichardt, Ch.S. (2000). Métodos cualitativos y cuantitativos en investigación evaluativa ( $2^{\mathrm{a}}$ ed.). Madrid: Morata.

Denzin, N.K. y Lincoln, I.S. (Coords.) (2012). El campo de la investigación cualitativa. Manual de investigación cualitativa (Nol. I). Barcelona: Gedisa.

Dussel, I. (2015). La incorporación de TIC en la formación docente de los países del MERCOSUR: estudios comparados sobre políticas e instituciones ( $1^{\mathrm{a}}$ ed.). Buenos Aires: Teseo.

Ertmer, P.A. (2005). Teacher pedagogical beliefs: The final frontier in our quest for technology integration. Educational Technology, Research and Development, 53(4), 25-39.

Ertmer, P.A. y Ottenbreit-Leftwich, A.T. (2010). Teacher Technology Change: How Knowledge, Confidence, Beliefs, and Culture Intersect. Journal of Research on Technology in Education, 42(3), 255-284. http://dx.doi. org/10.1080/15391523. 2010. -10782551

Flick, U. (2014). La gestión de la calidad de la investigación cualitativa. Madrid: Morata.

Flick, U. (2007). Introducción a la investigación cualitativa. Madrid: Morata.

Fullan, M. (2012). Los nuevos significados del cambio en la educación ( $2^{\mathrm{a}}$ ed.). Barcelona: Octaedro.

Fullan, M. y Donnelly, K. (2013). Alive in the swamp. Assessing digital innovations in Education. London: Nesta. Recuperado de https://www. nesta.org.uk/sites/ default/files/alive_in_the_ swamp.pdf

Gómez, C., Ortuño, J. y Molina, S. (2014). Aprender a pensar históricamente. Retos para la historia en el siglo XXI. Tempo \& Argumento. Revista de Historia do Tempo Presente. (11), 5-27. http:// dx.doi.org/10.5965/2175180306112014005

Guba, E.G. y Lincoln, Y.S. (2012). Controversias paradigmáticas, contradicciones y confluencias emergentes. En Denzin, N. K. \& Lincoln, I. S.
(Coords.) (2012). El campo de la investigación cualitativa. Manual de investigación cualitativa (Vol. 2), pp. 38-78. Barcelona: Gedisa.

Hepp, P. (2015). Desafíos de las políticas de integración de tecnología en la formación inicial y continua de los docentes. En Poggi, M. (Coord.) Mejorar los aprendizajes en la enseñanza obligatoria: políticas y actores. IIPE/ UNESCO (pp. 195-214). Recuperado de http:// unesdoc.unesco.org/images/0023/002349/ 234977s.pdf

Koehler, M.S., Mishra, P. y Cain, W. (2015). ¿Qué son los saberes tecnológicos y pedagógicos del contenido (TPACK)? Virtualidad, Educación y Ciencia, 6(10), 9-23. Recuperado de https:// revistas.unc.edu.ar/index.php/vesc/article/ view/ 11552/11983

Kvale, S. (2011). Las entrevistas en Investigación Cualitativa. Madrid: Morata.

Marcelo García, C. (2013). Las tecnologías para la innovación y la práctica docente. Revista Brasileira de Educaçao, 18(52), 25-47. Recuperado de http://www.scielo.br/pdf/ rbedu/v18n52/03.pdf

Maxwell, J. A. (1996). Diseño de investigación cualitativa. Un enfoque interactivo. Métodos de investigación social aplicados. [Traducido al español de Qualitative research design. An interactive approach. Applied social research methods]. California: Sage. Recuperado de http://www.catedras.fsoc.uba.ar/guemure/ bibliografia/Maxwell1.pdf

Mejía Navarrete, J. (2011). Problemas centrales del análisis de datos cualitativos. Revista Latinoamericana de Metodología de la Investigación Social, 1, 47-60. Recuperado de http://www.relmis.com.ar/ojs/index.php/ relmis/article/view/11/1

Mejía Navarrete, J. (2000). El muestreo en la investigación cualitativa. Investigaciones sociales, 4(5), 165-180. Recuperado de https://revistasinvestigacion.unmsm.edu.pe/ index.php/sociales/article/view/6851/6062 
Miles, M.B. y Huberman, A.M. (1994). Qualitative data analysis. An expanded sourcebook, (2nd ed.). Thousand Oaks, California: Sage.

Moral, C. (2006). Criterios de validez en la investigación cualitativa actual. Revista de Investigación Educativa, (24)1, 147-164. Recuperado de https://dialnet.unirioja.es/ servlet/articulo?codigo=2262155

Pagès Blanch, J. (1999). El tiempo histórico: ¿qué sabemos sobre su enseñanza y su aprendizaje? Análisis y valoración de los resultados de algunas investigaciones. Aspectos didácticos de Ciencias Sociales. Historia, 13, 241-278.

Pagès Blanch, J. y Santisteban Fernández, A. (2013). Una mirada al pasado y un proyecto de futuro. En Pagès Blanch, J. \& Santisteban Fernández, A. (Eds.) Una mirada al pasado y un proyecto de futuro. Investigación e innovación en didáctica de las ciencias sociales 1 (pp. 17-39). Barcelona: Asociación Universitaria de Profesorado de Didáctica de las Ciencias Sociales.

Santisteban, A. (2007). Una investigación sobre cómo se aprende a enseñar el tiempo histórico. Enseñanza de las Ciencias Sociales. Revista de investigación, 6, 19-29.

Simons, H. (2011). El estudio de caso: teoría y práctica. Madrid: Morata.

Stake, R. (2010). Investigación con estudio de casos ( $2^{\mathrm{a}}$ ed.). Madrid: Morata.

Taylor, S. J. y Bogdan, R. (2000). Introducción a los métodos cualitativos de investigación. La búsqueda de significados. Buenos Aires: Paidós.

Vaillant, D. y Marcelo, C. (2015). El ABC y D de la Formación Docente. Madrid: Narcea.

Valles, M.S. (2003). Técnicas Cualitativas de Investigación Social. Reflexión metodológica y práctica profesional. Madrid: Síntesis.

Webb, M.E. y Cox, M. (2004). A review of pedagogy related to information and comunications technology. Technology, Pedagogy and Education, 13(3), 235-286. https://doi. org/10.1080/14759390400200183

Yuni, J. y Urbano, C. (2006). Técnicas para investigar 2. Recursos metodológicos para la preparación de proyectos de investigación. Córdoba: Brujas. 